\title{
Effets de substitution des engrais chimiques par Azolla pinnata en riziculture au Nord Bénin
}

\author{
Antoine Z. K. DJOGBEDE, Lambert Cloud HINVI et Emile Didier FIOGBE* \\ Unité de Recherche sur les Zones Humides URZH/FAST-UAC, Bénin. \\ *Auteur correspondant ; E-mail : edfiogbe@yahoo.fr
}

\section{RESUME}

La culture du riz au Bénin s'est accrue ces dernières années en raison, de l'importance que prend cette denrée alimentaire dans les ménages et la restauration collective. Cette culture se fait avec des quantités importantes d'engrais chimiques qui, créent des problèmes environnementaux tels que, la pollution des sols et des nappes phréatiques. Azolla, petite fougère aquatique utilisée en riziculture en Asie est, expérimentée dans une exploitation rizicole grâce à des essais de substitution à différentes doses d'engrais. Le dispositif d'évaluation utilisé est composé de blocs à 3 répétitions par traitement comportant 6 traitements. La fertilisation apportée comporte deux fractions : le NPK en fumure de fond au repiquage, et l'urée 30 jours après le repiquage pour les traitements 2, 3, 4 et 5 ; Azolla pour le traitement 1 et l'absence de fertilisation pour le traitement 6 qui est le traitement témoin. La densité de mise en place des plants de riz dans ce dispositif est aux écartements de $20 \mathrm{~cm}$ x $20 \mathrm{~cm}$ à raison de 3 plants par poquet sur une superficie de $5 \mathrm{~m}^{2}$ par répétition (essai) soit, $15 \mathrm{~m}^{2}$ par traitement. Les mesures de vingt plants fixés et numérotés par casier soit un total de 60 plants identifiés par traitement ont été effectuées sur des plants choisis au centre des casiers pour éviter l'effet de périphérie qui fait développer les plants périphériques mieux que les plants situés au centre des casiers. Les résultats montrent que, la masse de paddy et la masse de matière sèche (production de fourrage) sont significativement plus élevées en fertilisation avec Azolla pinnata qu'en fertilisation chimiques quelque soit la dose d'engrais chimique. L'utilisation de Azolla pinnata permet d'éviter la pollution de l'espace cultivé par les engrais chimiques et les pesticides.

(C) 2012 International Formulae Group. All rights reserved.

Mots clés: Engrais chimique: N P K et urée, substitution, Azolla, engrais vert, croissance, rendement.

\section{INTRODUCTION}

Le Bénin comme la plupart des pays africains est confronté au changement des mœurs alimentaires caractérisé, par une place de plus en plus importante qu'occupe la consommation du riz, jadis considéré comme aliment de fête. Cela a entrainé une augmentation de la production du riz au Bénin.
La production nationale du riz qui, ne représentait que $0,31 \%$ de la production ouest africaine qui est de l'ordre de 6136000 tonnes (FAO, 2001) depuis dix ans, connaît à l'heure actuelle une croissance significative (Verlinden et Soulé, 2003). La production du riz au Bénin a été multipliée par cinq entre 1990 et 2000. Elle est passée de 10.940 tonnes de paddy en 1990 à 52.441 tonnes de paddy en 2000 (MDR/DPP, 2000). En 2004, elle a 
atteint 64.151 tonnes (DPP/MAEP, 2004). La plus grande partie de cette production est concentrée dans les départements de l'Alibori (34\%), des Collines (32\%), de l'Atakora (16\%), du Borgou (8\%) et de la Donga (6\%). Les départements du Couffo, du Zou, du Mono, de l'Ouémé et des Plateaux contribuent faiblement à cette production (Adégbola et Singbo, 2005).

Pour relever le niveau de la production nationale et atteindre l'autosuffisance alimentaire dans ce secteur, des mesures d'aménagement de bas-fonds et des périmètres irrigués ont été prises. De même, des variétés plus performantes comme le riz NERICA ont été mises au point par la Recherche et mises à la disposition des riziculteurs. Ces variétés sont cultivées dans trois types d'écosystèmes : les bas-fonds, les périmètres irrigués et les plateaux en terre exondée. Cela fait appel à l'utilisation abusive des fertilisants et des herbicides qui, expose les sols et les nappes phréatiques à une pollution de plus en plus croissante comme c'en est, le cas à Banikoara pour le coton (Assigbe, 2003).

L'utilisation des intrants agricoles pollue l'environnement et augmente le prix de revient de la production du riz. En effet, ces engrais ne sont pas assez purifiés et renferment de nombreux métaux lourds et toxiques peu mobiles dans le sol. Dans les super phosphates, des taux d'impuretés sont de l'ordre de 7 à 32 ppm de Nickel, 66 à 243 ppm de chrome, 7 à 92 ppm de plomb et 50 à 1430 ppm de Zinc (Brohl et al., 1997). Ces substances peu mobiles, s'accumulent dans les horizons superficiels des sols en particulier, dans la couche cultivée où sont situées les racines de riz qui absorbent ces métaux et les concentrent dans leurs tissus. Les effets de ces concentrations sont entre autres, responsables de l'augmentation des risques de cancer chez les consommateurs de ces denrées. L'abus de ces engrais chimiques n'a pas que des inconvénients sur la santé de l'homme. Il provoque aussi des modifications de la structure physique des sols entraînant, une modification de la percolation, la capacité de rétention des ions de ces sols ainsi que leur capacité d'échange cationique (Sadiki et Hilali, 1992). Les herbicides quant à eux, une fois propagés, s'infiltrent dans le sol et les eaux polluant ainsi la nappe phréatique (Gaur, and Noraho (1995)

Ces engrais et herbicides, en dehors de leur prix d'achat élevé présentent donc, des dangers aussi bien sur la santé de l'Homme que sur la pollution de l'environnement.

Pour remédier à ces différents maux, les Asiatiques depuis l'antiquité font appel à l'utilisation de Azolla comme engrais vert dans leur riziculture (Rakotonasolo, 1988). Cette pratique culturale est également introduite à Madagascar et en Afrique du Sud pour les mêmes raisons (Rakotonasolo, 1988).

En effet, Azolla est une petite fougère aquatique capable de transformer l'azote atmosphérique $\left(\mathrm{N}_{2}\right)$ en ammoniac $\left(\mathrm{NH}_{3}\right)$ par le biais d'une symbiose héréditaire avec une cyanobactérie (Anabeana azolla). Cette bactérie est sous forme de filaments non ramifiée constituant les cellules végétatives dont certaines se différencient en hétérocystes (Van Hoove, 1983). Ces hétérocystes sont des sites exclusifs de la fixation de l'azote atmosphérique (Reynaud, 1986). En riziculture, Azolla pinnata permet la fertilisation du sol, donc l'augmentation de la productivité du riz grâce à sa richesse en azote. Selon Anonyme (1998), un kilogramme de Azolla frais de bonne qualité peut fournir l'élément fertilisant apporté par 4 grammes d'urée commerciale. C'est fort de cela que ce travail de recherche visant une riziculture respectueuse de l'environnement à travers la substitution des engrais chimiques par Azolla pinnata dans des exploitations au Nord du Bénin a été initié.

\section{MATERIEL ET METHODES}

Une variété de riz Adny 11, ayant un rendement potentiel de 5 à 7,5 tonnes à l'hectare et un rendement réel de 4 à 6 tonnes à l'hectare, a été évaluée dans une station de recherche située dans les bas-fonds de Bamora/Banikanni à Parakou. Il a une très bonne réponse à la fumure avec un cycle 
végétatif de 105 à 110 jours. La ville de Parackou est caractérisée par des précipitations unimodales et une pluviométrie annuelle de $1100 \mathrm{~mm}$, une saison pluvieuse qui va d'Avril à Octobre (DPP/MAEP. 2004). Le sol du site est formé de sable lagunaire et situé à N $09^{\circ} 18^{\prime}$ 51,4' E $002^{\circ} 38^{\prime} 47^{\prime}$ ' Alt. 334 m (DPP/MAEP. 2004)

A. pinnata var africana, est une fougère aquatique productrice d'ammoniaque à partir de l'azote atmosphérique. Elle provient du laboratoire de l'Unité de Recherche sur les Zones Humides et est utilisée en Asie et au Madagascar en riziculture comme fertilisant biologique. Elle est aussi utilisée comme fertilisant organique.

Une pépinière de plants de riz a été mise en place auparavant afin d'avoir des jeunes plants de riz qui seront repiqués plus tard entre 25 et 30 jours des semis. Ces plantules ont été déterrées, puis repiquées soigneusement et suivies par quinzaine.

Le dispositif d'évaluation est un bloc de 3 répétitions par traitement et comporte 6 traitements. Les plants de riz ont été mis en charge aux écartements de $20 \mathrm{~cm}$ x $20 \mathrm{~cm}$ à raison de 3 brins par poquet sur une superficie de $5 \mathrm{~m}^{2}$ par répétition (essai) soit $15 \mathrm{~m}^{2}$ par traitement.

La fertilisation a été apportée en deux fractions : le NPK en fumure de fond au repiquage et l'urée 30 jours après le repiquage pour les traitements 2, 3, 4 et 5, Azolla pour le traitement 1 et l'absence de fertilisation pour le traitement 6 qui est le traitement témoin (Tableau 1).

\section{Méthode de collecte des données}

Elle consiste à recueillir les données suivantes :

- Hauteur des plants de riz par quinzaine ;

- Nombre de feuilles par poquet par quinzaine ;
- Nombre de thalles par poquet par quinzaine ;

- Nombre de panicules par poquet par quinzaine ;

- Poids moyen de paille et de paddy, et la masse de matière sèche de riz par casier et par essai en fin de récolte.

La mesure de la hauteur des plants de riz s'est faite par un mètre à ruban. Les mesures effectuées se rapportent à vingt plants fixés et numérotés par casier soit 60 plants identifiés par traitement. Ces plants ont été choisis au centre des casiers pour éviter l'effet de périphérie qui fait développer les plants périphériques mieux que les plants de la base jusqu'à l'extrémité de la feuille la plus longue, situées au centre des casiers.

Les données ont été encodées à l'aide du logiciel Excel ; les différentes analyses ont été faites à l'aide des logiciels SPSS et Minitab. Le traitement des données s'est basé sur le test d'analyse de variance et de comparaison multiple des moyennes suivant le dispositif $\mathrm{du}$ Tableau 3. Le dispositif expérimental est un groupe randomisé. Les valeurs de Fisher et de probabilité pour que l'hypothèse nulle des effets soit acceptée ou non, ont permis de conclure l'existence d'une différence significative ou non au seuil de probabilité de 5\%. Ce test d'analyse de variance (ANOVA subject-effect) nous a permis de voir la signification de l'effet des traitements sur les paramètres de croissance et de rendement.

Suite aux analyses de variances, il a été fait le test de comparaison multiple de moyenne, le test de Student et de NewmanKeuls des hauteurs, du nombre de feuilles, du nombre de thalles, du nombre de panicules et des différents rendements (rendement grain, rendement en paille et la masse de matière sèche). 


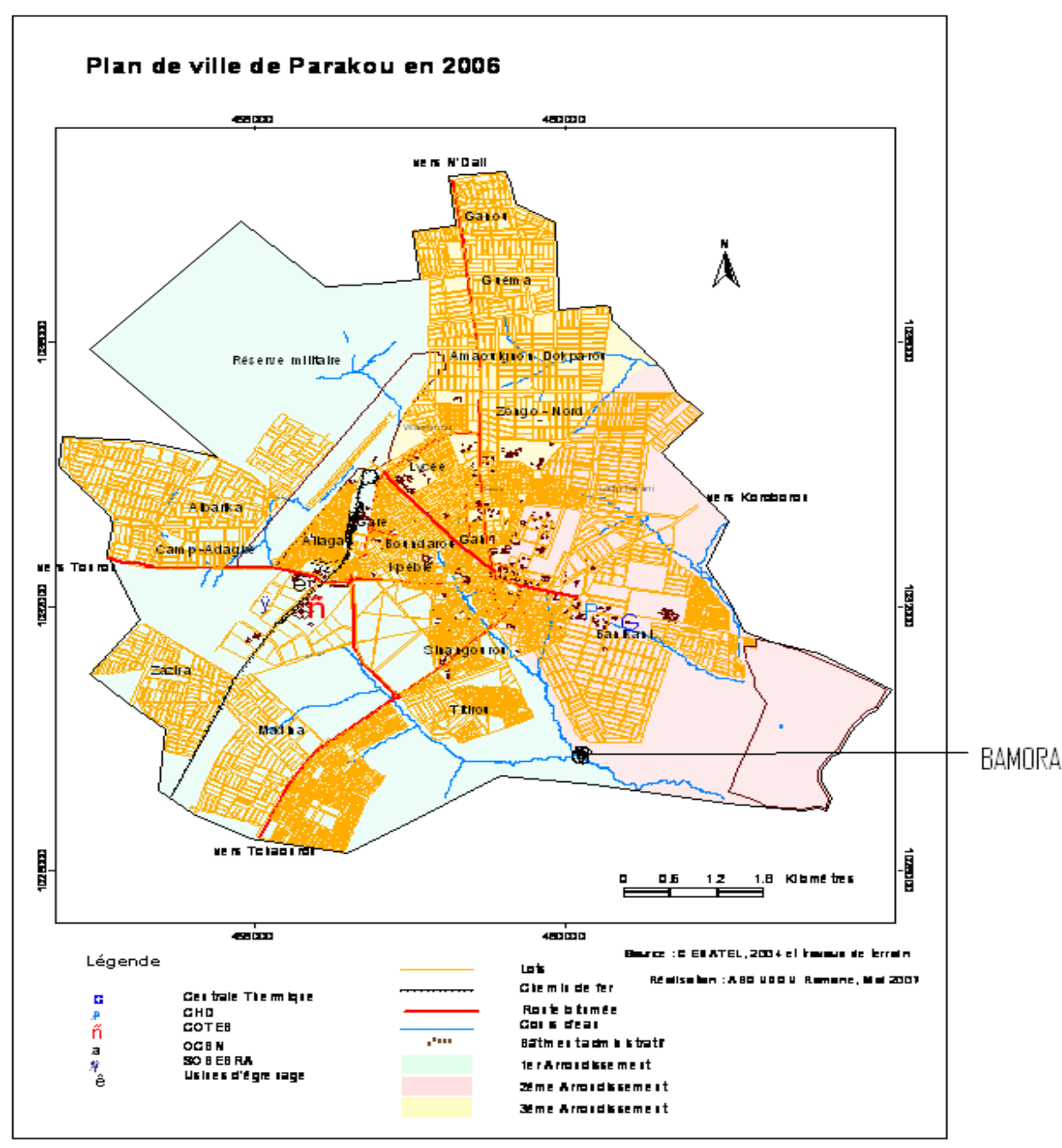

Figure 1 : Plan de la ville de Parakou.

\section{RESULTATS}

Effet de substitution des engrais chimiques par A. pinnata sur la croissance du riz

Effet de substitution des engrais chimiques par $A$. pinnata sur la croissance en hauteur $(\mathrm{cm})$ du riz.

L'analyse de variance de la croissance en hauteur des plants de riz révèle que l'effet de la fertilisation sur la hauteur des plants est significatif au seuil de $5 \%$ à partir de la première quinzaine pour tous les traitements. La hauteur des plants est plus élevée en fertilisation avec $A$. pinnata qu'en fertilisation chimique quelque soit la dose d'engrais chimique selon le test de Student Newman et
Keuls (Tableau 2). L'effet de la fertilisation sur la hauteur des plants de riz de différents traitements montre que le traitement 4 donne une hauteur plus élevée que les autres traitements à engrais chimiques et le témoin.

L'effet de substitution des engrais chimiques par $A$. pinnata en riziculture révèle que la croissance en hauteur des plants de riz diminue quand la quantité d'engrais chimique baisse à partir $100 \mathrm{~kg}$ de NPK et 50 kg d'Urée par hectare apportée devient de plus en plus faible lorsque cette même quantité d'engrais chimique augmente. Cette croissance en fertilisation organique de A. pinnata est plus élevée. La croissance est continue mais 
devient faible à partir de la cinquième quinzaine.

Effet de substitution des engrais chimiques par A. pinnata sur la foliation

L'analyse de variance de la croissance du nombre de feuilles par poquet des plants de riz révèle que l'effet de la fertilisation sur le nombre de feuilles émises par les plants est significatif au seuil de $5 \%$ dès la première quinzaine. Le nombre de feuilles émises par les plants est plus élevé en fertilisation avec A. pinnata que le nombre de feuilles portées par les plants en fertilisation chimiques quelque soit la dose d'engrais chimique selon le test de Student Newman et Keuls comme l'indique le Tableau 3.

Trois quinzaines après le repiquage, l'effet de la fertilisation sur le nombre de feuilles émises par les plants de riz de différents traitements montre que le traitement 4 donne une foliation plus élevée que les autres traitements à engrais chimiques.

La substitution des engrais par $A$. pinnata donne une foliation plus accrue que la foliation en fertilisant chimique. Cette formation de feuilles culmine et s'arrête cependant dès la quatrième quinzaine pour le traitement avec A. pinnata et à partir de la cinquième quinzaine pour les autres traitements.

Effet de substitution des engrais chimiques par Azolla pinnata sur la mise en place des thalles

L'analyse de variance de la croissance du nombre de thalles par poquet des plants de riz révèle que l'effet de la fertilisation sur le nombre de thalles émis par les plants est significatif au seuil de $5 \%$ à partir de la deuxième quinzaine pour tous les traitements. Le nombre de thalles émis par les plants est plus élevé en fertilisation avec $A$. pinnata que le nombre de thalles portés par les plants en fertilisation chimiques quelque soit la dose d'engrais chimique selon le test de Student
Newman et Keuls comme l'indique le Tableau 4.

Dès la deuxième quinzaine après repiquage, l'effet de la fertilisation sur le nombre de thalles émis par les plants de riz de différents traitements montre que le traitement 4 donne une mise en place de thalles plus élevée que les autres traitements à engrais chimiques.

La mise en place des thalles qui commence à la première quinzaine est mieux faite en présence de $A$. pinnata et s'achève à la cinquième quinzaine. De là se déroule la régression des thalles chétifs.

Effet de substitution des engrais chimiques par A. pinnata sur la floraison et la fructification

L'analyse de variance de la croissance du nombre de panicules par poquet des plants de riz révèle que l'effet de la fertilisation sur le nombre de panicules émis par les plants est significatif au seuil de $5 \%$ à partir de la quatrième quinzaine pour tous les traitements. Le nombre de panicules émis par les plants est plus élevé en fertilisation avec Azolla que le nombre de panicules portées par les plants en fertilisation chimiques quelque soit la dose d'engrais chimique selon le test de Student Newman et Keuls comme l'indique le Tableau 5.

L'effet de la fertilisation sur la floraison et la fructification des plants est significatif au seuil de $5 \%$ dès la quatrième quinzaine après repiquage selon le test de Student Newman et Keuls, et atteint son maximum à la cinquième quinzaine pour le traitement avec A. pinnata. Les autres traitements continuent d'augmenter légèrement le nombre de panicules.

L'effet de la fertilisation sur la floraison et la fructification des plants est significatif au seuil de $5 \%$ de la quatrième quinzaine après repiquage selon le test de Student Newman et Keuls. Il existe une différence significative selon les traitements et 
selon le temps. Les panicules sont les précurseurs des grains du riz et annoncent la bonne récolte. En fertilisation avec Azolla, les panicules sont plus nombreuses et à partir de la cinquième quinzaine, les panicules malformées tombent.

Effet de substitution des engrais chimiques par $A$ pinnata sur les rendements à la récolte

Les rendements sont donnés par :

- le rendement graine qui représente la masse du riz en paddy à $14 \%$ d'humidité et est estimé en tonnes par hectare ;

- le rendement paille qui représente la masse de paille récoltée (feuilles et chaumes) par traitement. Il est exprimé en tonnes par hectare; la masse de matière sèche qui est le cumul des masses de paddy et de paille, elle est exprimée en tonnes par hectare et,

- l'indice IH qui représente le rapport du rendement graine à la masse de matière sèche Il permet d'apprécier la rentabilité et permet d'orienter si l'on doit utiliser Azolla. comme fertilisant pour produire des plantes fourragères ou pour produire des paddy de riz.

L'analyse de variance des rendements des traitements révèle que l'effet de la fertilisation sur les rendements donnés par les traitements est significatif au seuil de $5 \%$. La masse de paddy et la masse de matière sèche sont plus élevées en fertilisation avec Azolla qu'en fertilisation chimiques quelque soit la dose d'engrais chimique selon le test de Student Newman et Keuls comme l'indique le Tableau 6.

Cela montre que le rendement grain du traitement 1 à Azolla est nettement supérieur aux autres traitements. Le rendement grain montre une différence significative entre les six (6) traitements au seuil de 5\% selon le test de Student Newman et Keuls. Par ordre chronologique on a, le traitement 1 avec
Azolla qui donne un rendement plus élevé suivi de celui des traitements $4,3,2,5$ et 6 .

Le rendement paille montre l'existence de quatre groupes homogènes bien que la production de paille du traitement 3 soit la plus élevée. La production de paille est considérable en traitement 1 avec Azolla mais est très élevée avec le traitement 3 qui donne le meilleur rendement paille. Le rendement paille montre une différence significative entre les quatre (4) traitements (groupe homogène) au seuil de $5 \%$ selon le test de Student Newman et Keuls.

L'analyse de variance au seuil de 5\% montre que l'effet de substitution des engrais chimiques par A. pinnata est significatif. Le rendement en masse de matière sèche montre que la productivité primaire est significativement plus élevée avec le traitement 1 avec $A$ pinnata que les autres traitements $(\mathrm{P}<0,05)$. Le traitement avec $A$. pinnata donne une masse de matière sèche plus élevée que les autres traitements. Il est suivi du traitement 4 , puis respectivement des traitements $3,2,5$ et 6 .

- L'indice IH est plus élevé en traitement 1 à $A$. pinnata que les autres traitements.

- L'indice IH aussi donne une différence significative au seuil de 5\% selon le test de Student Newman et Keuls. Il est plus fort pour le traitement 1 suivi respectivement des traitements 2, 3, 4, 5 et en fin du 6.

Cet indice permet d'apprécier lequel des traitements est plus indiqué pour le fourrage ou pour la production des grains de riz.

De tous ces résultats, on constate que les rendements en grain, et en masse de matière sèche (la productivité primaire) sont plus élevés lorsque les engrais chimiques sont substitués par A. pinnata. Dans ces conditions la production de la paille est significative et l'indice IH est aussi plus élevé. 
A Z. K. DJOGBEDE et al. / Int. J. Biol. Chem. Sci. 6(6): 3027-3044, 2012

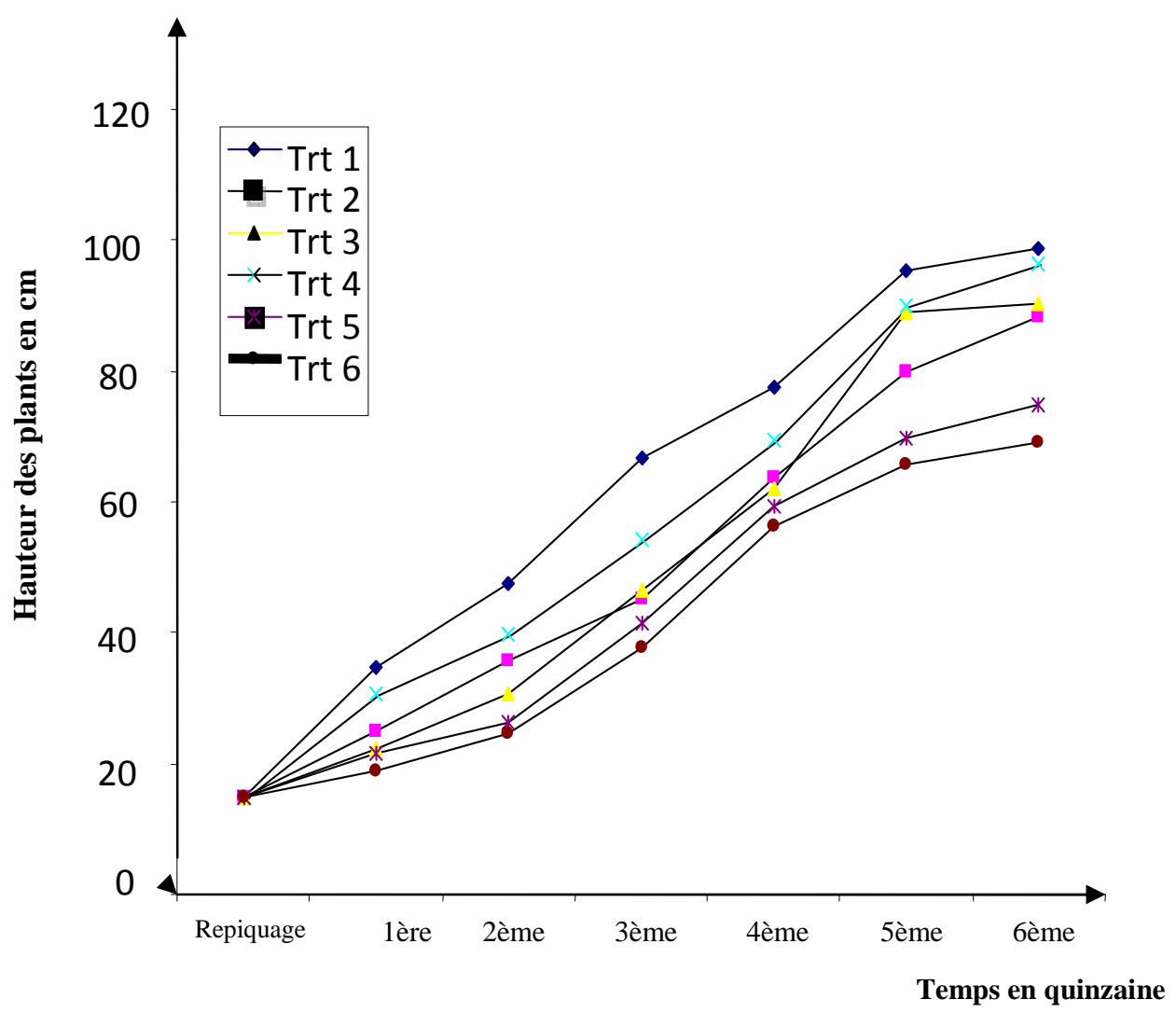

Figure 2: Effet de substitution des engrais par Azolla pinnata sur de la hauteur des plants du riz. 
A Z. K. DJOGBEDE et al. / Int. J. Biol. Chem. Sci. 6(6): 3027-3044, 2012

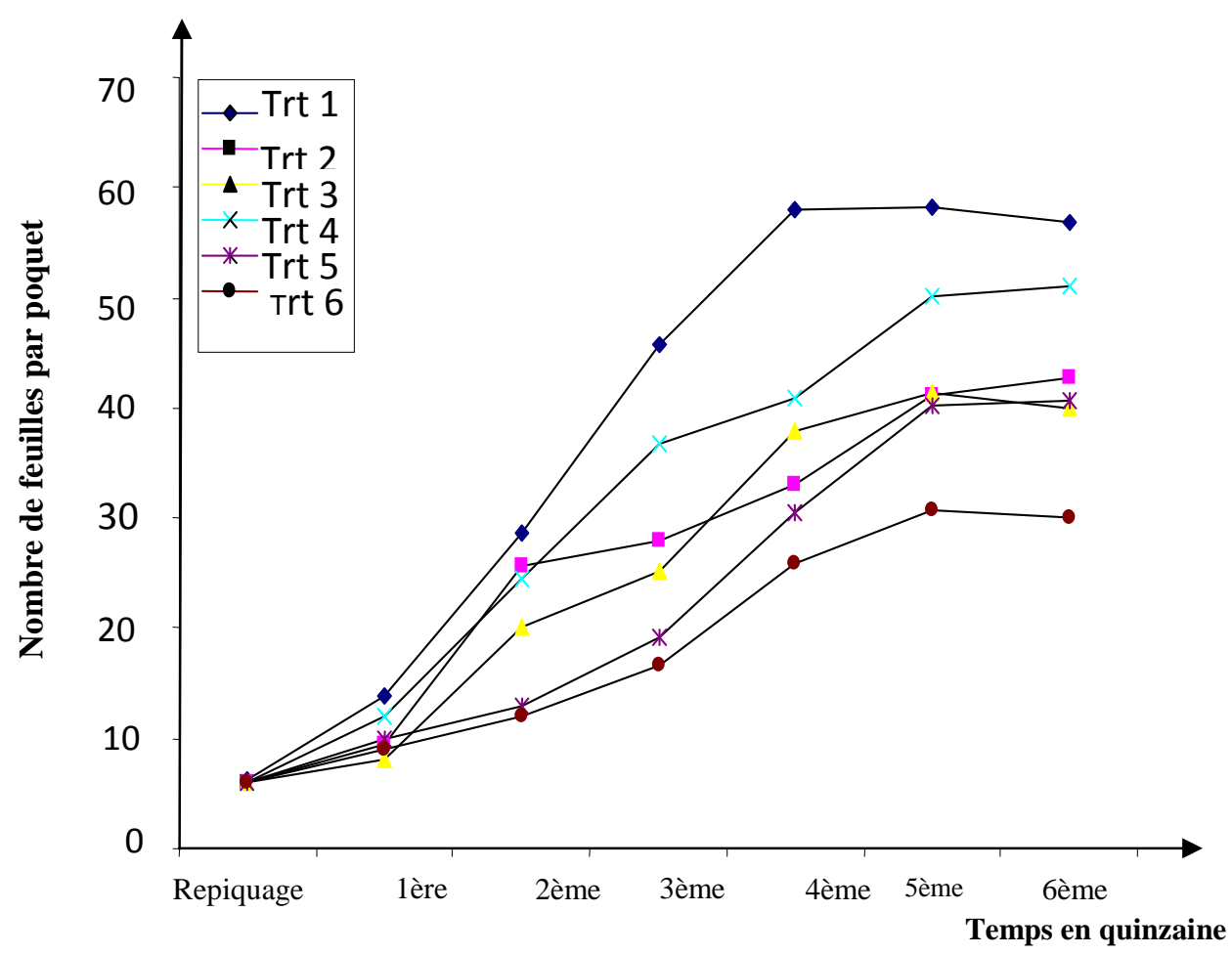

Figure 3 : Effet de substitution des engrais chimiques par Azolla pinnata sur la foliation. 
A Z. K. DJOGBEDE et al. / Int. J. Biol. Chem. Sci. 6(6): 3027-3044, 2012

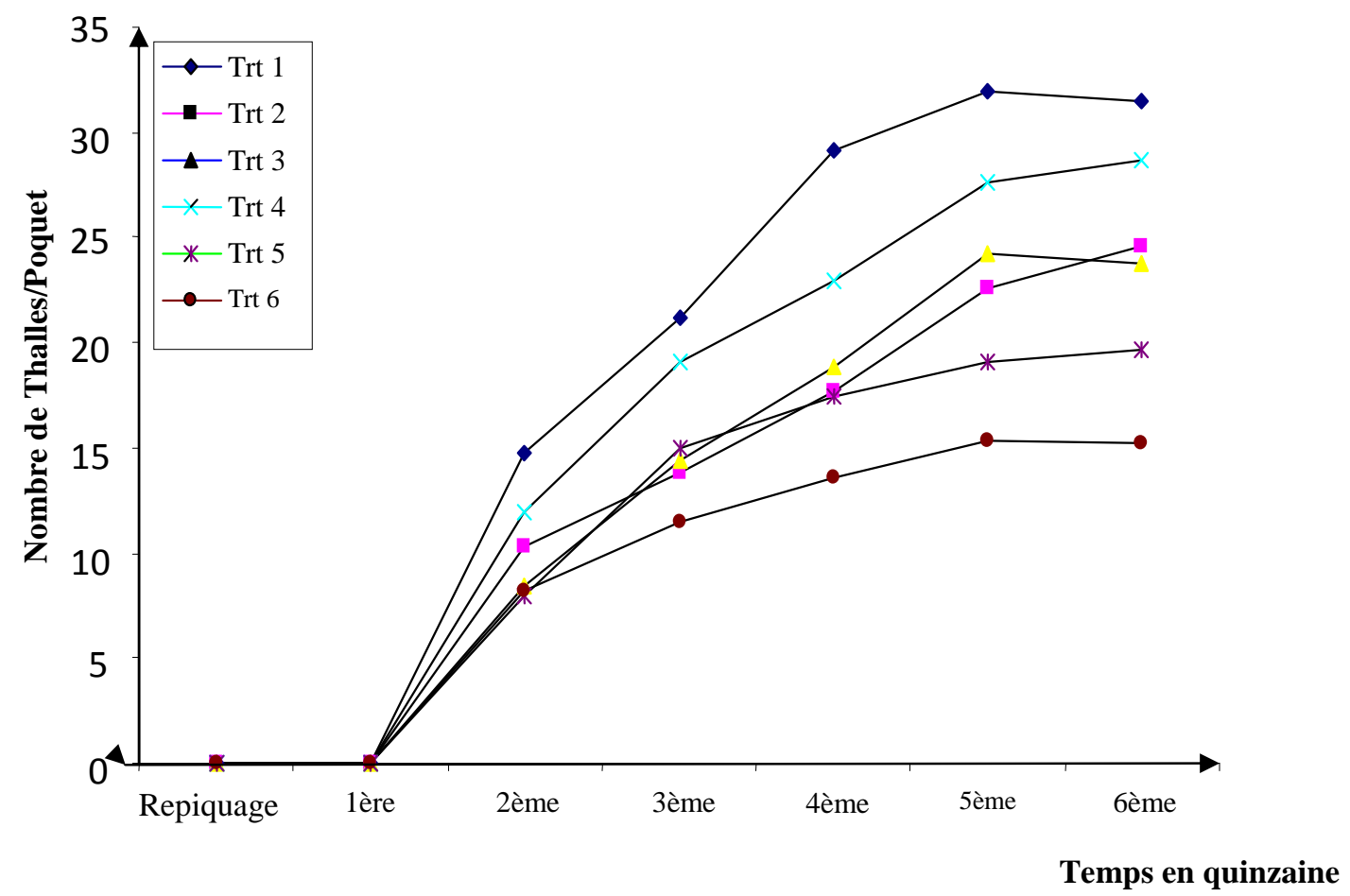

Figure 4: Effet de substitution des engrais chimiques par Azolla pinnata sur la mise en place des thalles. 


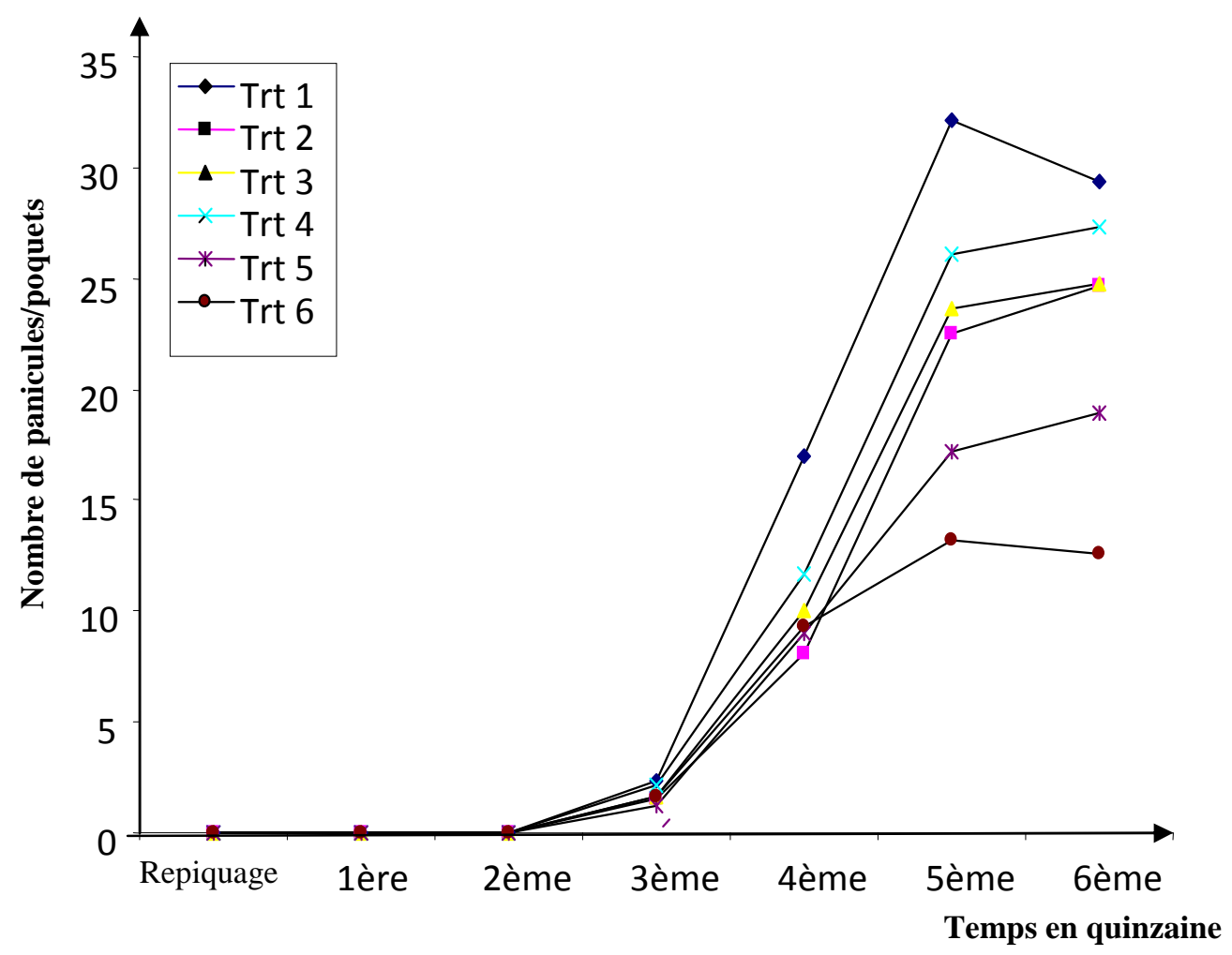

Figure 5: Effet de substitution des engrais chimiques par Azolla pinnata sur la floraison et la fructification.

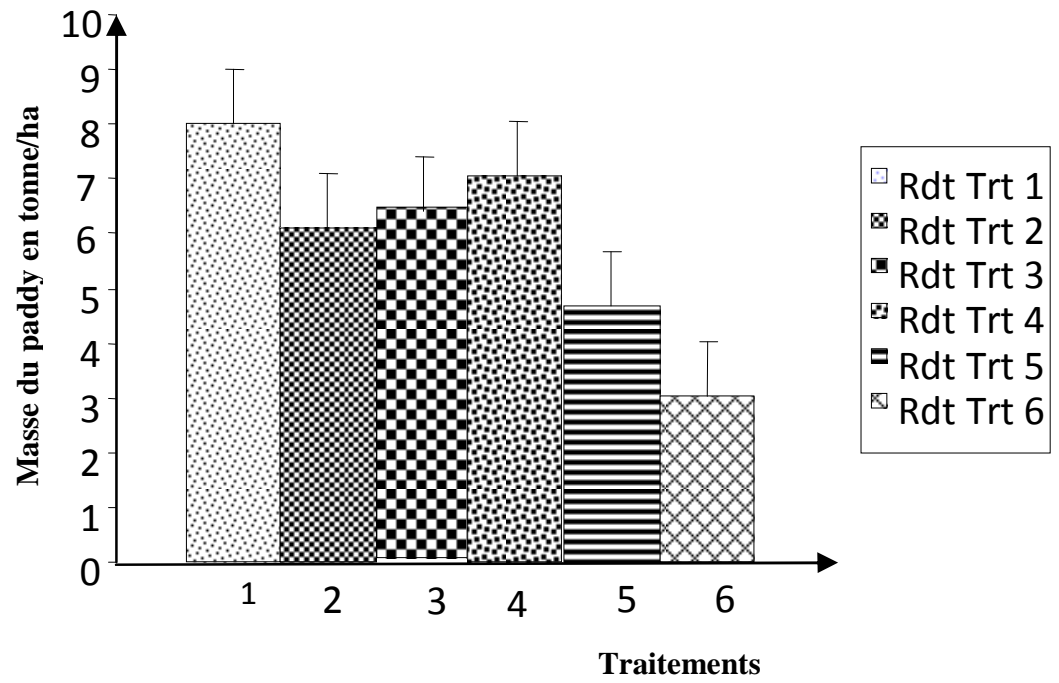

Figure 6: Effet de substitution des engrais chimiques par Azolla pinnata sur le rendement en grain (paddy). 


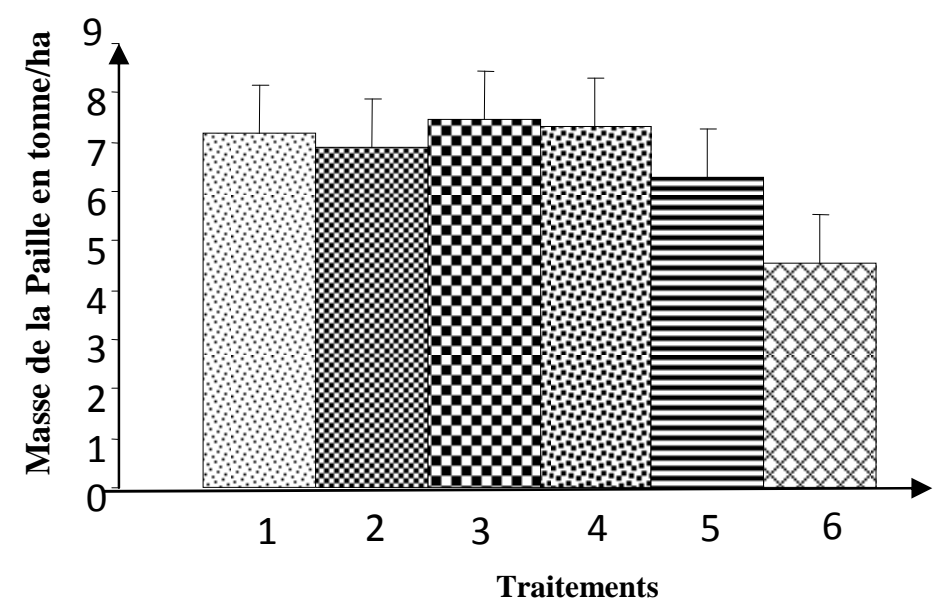

Rdt Trt 1

${ }^{\circ} \operatorname{Rdt}$ Trt 2

Rdt Trt 3

Rdt Trt 4

${ }^{\Xi} \mathrm{Rdt}$ Trt 5

${ }^{\otimes}$ Rdt Trt 6

Figure 7: Effet de substitution des engrais chimiques par Azolla pinnata sur le rendement en paille du riz.

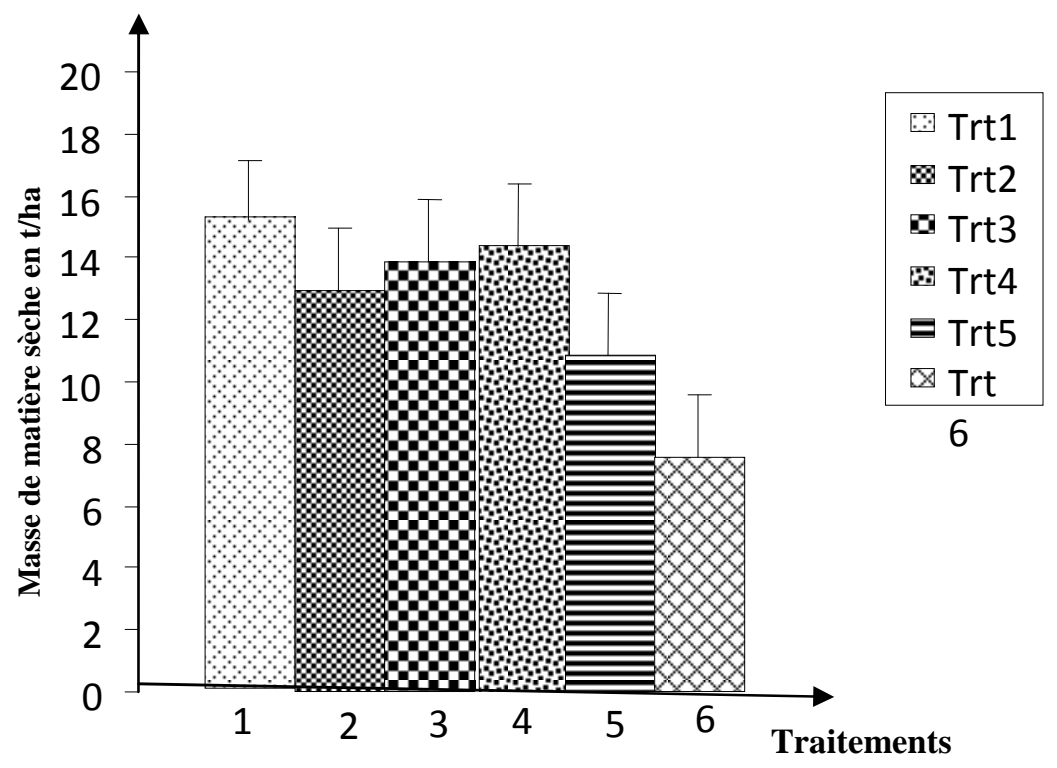

Figure 8: Effet de substitution des engrais chimiques par Azolla pinnata sur le rendement en matière sèche (Paille +Grain). 


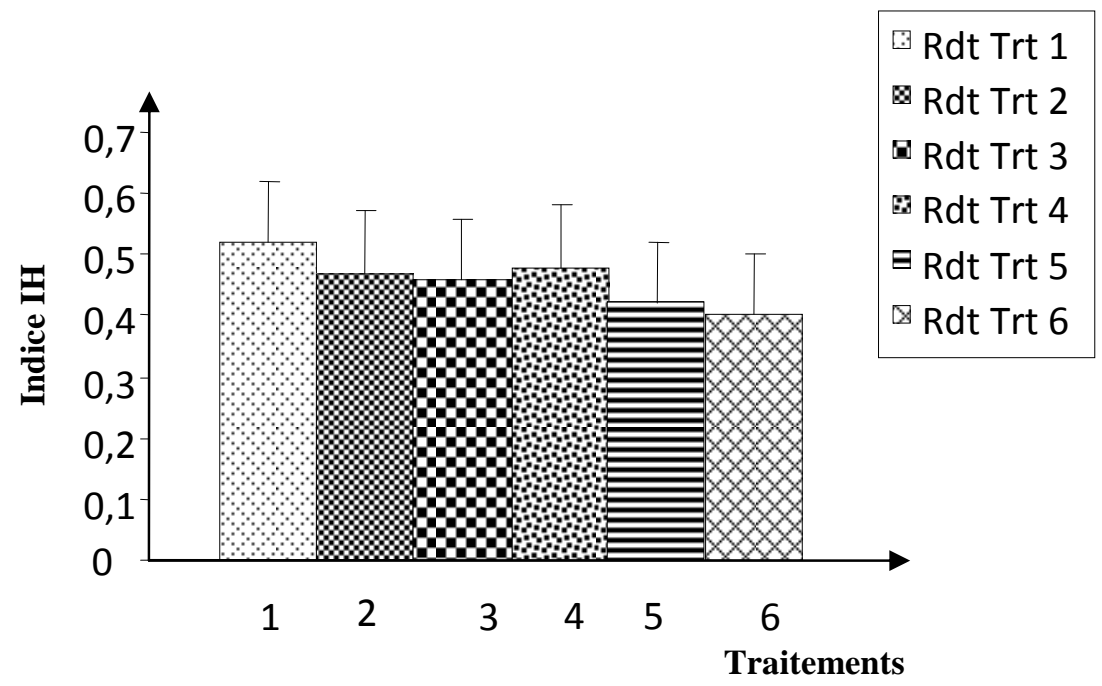

Figure 9: Effet de substitution des engrais chimiques par Azolla pinnata sur le rendement en indice IH.

Tableau 1: Fertilisation des traitements.

\begin{tabular}{lccc}
\hline Traitements & NPK & Urée & Azolla frais \\
\hline 1 & $0 \mathrm{~kg} / \mathrm{ha}$ & $0 \mathrm{~kg} / \mathrm{ha}$ & 3 tonnes $/ \mathrm{ha}$ \\
2 & $200 \mathrm{~kg} / \mathrm{ha}$ & $100 \mathrm{~kg} / \mathrm{ha}$ & $0 \mathrm{~kg} / \mathrm{ha}$ \\
3 & $150 \mathrm{~kg} / \mathrm{ha}$ & $75 \mathrm{~kg} / \mathrm{ha}$ & $0 \mathrm{~kg} / \mathrm{ha}$ \\
4 & $100 \mathrm{~kg} / \mathrm{ha}$ & $50 \mathrm{~kg} / \mathrm{ha}$ & $0 \mathrm{~kg} / \mathrm{ha}$ \\
5 & $50 \mathrm{~kg} / \mathrm{ha}$ & $25 \mathrm{~kg} / \mathrm{ha}$ & $0 \mathrm{~kg} / \mathrm{ha}$ \\
6 & $0 \mathrm{~kg} / \mathrm{ha}$ & $0 \mathrm{~kg} / \mathrm{ha}$ & $0 \mathrm{~kg} / \mathrm{ha}$ \\
\hline
\end{tabular}

Tableau 2: Hauteurs des plants de riz.

\begin{tabular}{|c|c|c|c|c|c|c|c|}
\hline \multirow[t]{2}{*}{ Traitements } & \multicolumn{7}{|c|}{ Temps en quinzaine } \\
\hline & $\mathbf{0}$ & 1 & 2 & 3 & 4 & 5 & 6 \\
\hline 1 & $14,72 \mathrm{a}$ & $34,75 \mathrm{e}$ & $47,63 f$ & $66,67 f$ & $77,40 \mathrm{f}$ & $95,48 \mathrm{e}$ & $98,67 f$ \\
\hline 2 & $14,88 \mathrm{a}$ & $25,10 \mathrm{c}$ & $35,75 d$ & $45,07 \mathrm{c}$ & $63,68 d$ & $79,85 \mathrm{c}$ & $88,42 \mathrm{c}$ \\
\hline 3 & $14,73 \mathrm{a}$ & $22,32 b$ & $30,55 \mathrm{c}$ & $46,67 d$ & $61,87 \mathrm{c}$ & $89,00 \mathrm{~d}$ & $90,42 d$ \\
\hline 4 & $14,93 \mathrm{a}$ & $30,52 d$ & $39,87 \mathrm{e}$ & $54,28 \mathrm{e}$ & $69,42 \mathrm{e}$ & $90,17 d$ & $96,42 \mathrm{e}$ \\
\hline 5 & $14,92 \mathrm{a}$ & $21,73 b$ & $26,27 b$ & $41,41 \mathrm{~b}$ & $59,35 b$ & $69,70 \mathrm{~b}$ & $74,67 b$ \\
\hline 6 & $14,95 \mathrm{a}$ & $18,97 \mathrm{a}$ & $24,77 \mathrm{a}$ & $37,85 \mathrm{a}$ & $56,30 \mathrm{a}$ & $65,57 \mathrm{a}$ & $68,93 \mathrm{a}$ \\
\hline
\end{tabular}


Tableau 3: Evolution du nombre de feuilles par poquet.

\begin{tabular}{lccccccc}
\hline & \multicolumn{7}{c}{ Temps (quinzaine) } \\
\cline { 2 - 8 } Traitement & $\mathbf{0}$ & $\mathbf{1}$ & $\mathbf{2}$ & $\mathbf{3}$ & $\mathbf{4}$ & $\mathbf{5}$ & $\mathbf{6}$ \\
\hline 1 & $6,13 \mathrm{~b}$ & $13,75 \mathrm{e}$ & $28,67 \mathrm{e}$ & $45,70 \mathrm{f}$ & $57,93 \mathrm{f}$ & $58,22 \mathrm{~d}$ & $56,88 \mathrm{e}$ \\
2 & $6,07 \mathrm{ab}$ & $9,53 \mathrm{bc}$ & $25,63 \mathrm{~d}$ & $28,03 \mathrm{~d}$ & $33,13 \mathrm{c}$ & $41,15 \mathrm{~b}$ & $42,72 \mathrm{c}$ \\
3 & $6,00 \mathrm{a}$ & $7,98 \mathrm{a}$ & $20,07 \mathrm{~b}$ & $25,07 \mathrm{c}$ & $37,93 \mathrm{~d}$ & $41,27 \mathrm{~b}$ & $40,07 \mathrm{~b}$ \\
4 & $6,05 \mathrm{ab}$ & $12,02 \mathrm{~d}$ & $24,33 \mathrm{c}$ & $36,73 \mathrm{e}$ & $40,97 \mathrm{e}$ & $50,22 \mathrm{c}$ & $51,08 \mathrm{~d}$ \\
5 & $6,03 \mathrm{ab}$ & $10,02 \mathrm{c}$ & $12,85 \mathrm{a}$ & $19,16 \mathrm{~b}$ & $30,38 \mathrm{~b}$ & $40,10 \mathrm{~b}$ & $40,65 \mathrm{~b}$ \\
6 & $6,03 \mathrm{ab}$ & $9,05 \mathrm{~b}$ & $11,95 \mathrm{a}$ & $16,53 \mathrm{a}$ & $25,83 \mathrm{a}$ & $30,68 \mathrm{a}$ & $29,97 \mathrm{a}$ \\
\hline
\end{tabular}

Tableau 4: Evolution du nombre de thalles par poquet.

\begin{tabular}{lccccccc}
\hline Traitement & \multicolumn{7}{c}{ Temps (quinzaine) } \\
\cline { 2 - 8 } & $\mathbf{0}$ & $\mathbf{1}$ & $\mathbf{2}$ & $\mathbf{3}$ & $\mathbf{4}$ & $\mathbf{5}$ & $\mathbf{6}$ \\
\hline 1 & 0 & 0 & $14,78 \mathrm{~d}$ & $21,18 \mathrm{e}$ & $29,17 \mathrm{e}$ & $32,00 \mathrm{f}$ & $31,50 \mathrm{f}$ \\
2 & 0 & 0 & $10,33 \mathrm{~b}$ & $13,87 \mathrm{~b}$ & $17,72 \mathrm{~b}$ & $22,65 \mathrm{c}$ & $24,57 \mathrm{~d}$ \\
3 & 0 & 0 & $8,43 \mathrm{a}$ & $14,41 \mathrm{bc}$ & $18,88 \mathrm{c}$ & $24,27 \mathrm{~d}$ & $23,73 \mathrm{c}$ \\
4 & 0 & 0 & $12,02 \mathrm{c}$ & $19,05 \mathrm{~d}$ & $23,00 \mathrm{~d}$ & $27,67 \mathrm{e}$ & $28,70 \mathrm{e}$ \\
5 & 0 & 0 & $8,00 \mathrm{a}$ & $15,00 \mathrm{c}$ & $17,42 \mathrm{~b}$ & $19,05 \mathrm{~b}$ & $19,70 \mathrm{~b}$ \\
6 & 0 & 0 & $8,14 \mathrm{a}$ & $11,50 \mathrm{a}$ & $13,62 \mathrm{a}$ & $15,37 \mathrm{a}$ & $15,23 \mathrm{a}$ \\
\hline
\end{tabular}

Dans les colonnes, les valeurs suivies d'une même lettre ne sont pas significativement différentes au seuil de probabilité de cinq pour cent (5\%) selon le test de Student Newman et Keuls.

Tableau 5: Evolution du nombre de panicules par poquet.

\begin{tabular}{lccccccc}
\hline Traitement & \multicolumn{7}{c}{ Temps en quinzaine } \\
\cline { 2 - 7 } & $\mathbf{0}$ & $\mathbf{1}$ & $\mathbf{2}$ & $\mathbf{3}$ & $\mathbf{4}$ & $\mathbf{5}$ & $\mathbf{6}$ \\
\hline 1 & 0 & 0 & 0 & $2,36 \mathrm{a}$ & $17,00 \mathrm{~d}$ & $32,13 \mathrm{f}$ & $29,42 \mathrm{e}$ \\
2 & 0 & 0 & 0 & $1,57 \mathrm{a}$ & $8,13 \mathrm{a}$ & $22,53 \mathrm{c}$ & $24,67 \mathrm{c}$ \\
3 & 0 & 0 & 0 & $1,67 \mathrm{a}$ & $10,02 \mathrm{~b}$ & $23,60 \mathrm{~d}$ & $24,73 \mathrm{c}$ \\
4 & 0 & 0 & 0 & $2,11 \mathrm{a}$ & $11,65 \mathrm{c}$ & $26,10 \mathrm{e}$ & $27,32 \mathrm{~d}$ \\
5 & 0 & 0 & 0 & $1,25 \mathrm{a}$ & $9,05 \mathrm{ab}$ & $17,15 \mathrm{~b}$ & $18,90 \mathrm{~b}$ \\
6 & 0 & 0 & 0 & $1,67 \mathrm{a}$ & $9,28 \mathrm{ab}$ & $13,17 \mathrm{a}$ & $12,55 \mathrm{a}$ \\
\hline \multicolumn{7}{c}{ Dans les colonnes, les valeurs suivies d'une même lettre ne sont pas significativement différentes au seuil de }
\end{tabular}


Tableau 6: Rendements à la récolte.

\begin{tabular}{ccccc}
\hline Traitement & Rendement G & Rendement P & MS & IH \\
\hline 1 & $8,02 \mathrm{f}$ & $7,16 \mathrm{~d}$ & $15,18 \mathrm{f}$ & $0,52 \mathrm{e}$ \\
2 & $6,09 \mathrm{c}$ & $6,86 \mathrm{c}$ & $12,96 \mathrm{c}$ & $0,47 \mathrm{c}$ \\
3 & $6,39 \mathrm{~d}$ & $7,45 \mathrm{~d}$ & $13,85 \mathrm{~d}$ & $0,46 \mathrm{c}$ \\
4 & $7,06 \mathrm{e}$ & $7,31 \mathrm{~d}$ & $14,38 \mathrm{e}$ & $0,48 \mathrm{~d}$ \\
5 & $4,66 \mathrm{~b}$ & $6,26 \mathrm{~b}$ & $10,86 \mathrm{~b}$ & $0,43 \mathrm{~b}$ \\
6 & $3,04 \mathrm{a}$ & $4,55 \mathrm{a}$ & $7,58 \mathrm{a}$ & $0,40 \mathrm{a}$
\end{tabular}

Dans les colonnes, les valeurs suivies d'une même lettre ne sont pas significativement différentes au seuil de probabilité de cinq pour cent (5\%) selon le test de Student Newman et Keuls.

\section{DISCUSSION}

Effet de substitution des engrais chimiques par A. pinnata sur la croissance plants de riz

De cette étude, il ressort que la croissance sous fertilisation organique de Azolla est plus élevée qu'en fertilisation chimique minérale et la différence est significative, de même la croissance sous fertilisation chimique est plus élevée qu'en absence de fertilisation (témoin) et la différence est également significative $(P<0,05)$. En effet ces résultats obtenus sont conformes aux résultats de PSI/CORAF (1980) et ceux de IRAT et FAO/OMVS (1972) selon lesquels, le riz répond positivement aux apports d'azote. Pour Dobermann et al. (2000), la fertilisation en azote affecte tous les paramètres contribuant, à l'obtention d'un bon rendement (hauteur, nombre de feuilles, nombre de thalles nombre de panicules et nombre d'épillets par panicules). La fertilisation est alors nécessaire à la culture du riz ce qui est cependant contraire à la conclusion de Arf et al. (1996) qui, ont étudié certaines variétés de riz soumises à des doses variables d'azote par hectare et ont trouvé, que l'engrais n'a pas d'influence sur les caractéristiques agronomiques du riz. Mais on peut expliquer les réponses positives obtenues dans le cadre de cette étude par la pauvreté relative du sol de notre site expérimental par rapport à un sol de jachère ou forestier ou de zones humides naturellement riche en minéraux.

Le traitement 4 est celui qui a une croissance finale proche de la croissance du traitement1 sous fertilisation organique de Azolla. C'est le traitement dont la croissance est optimale en fertilisation chimique .Cela signifie que la fertilisation chimique de 100 $\mathrm{kg} / \mathrm{ha}$ de NPK et $50 \mathrm{~kg} / \mathrm{h}$ d'urée (Trt 4) est la fertilisation optimale ce qui n'est pas conforme aux travaux de Thio, Sawadogo, 2002 selon lesquels la dose optimale est la dose du traitement 3 (200 $\mathrm{kg}$ de NPK et 100 $\mathrm{kg}$ d'urée par hectare). On pourrait cependant expliquer cette différence par les conditions hydriques un peu difficiles au cours de l'expérimentation. Le témoin sans fertilisation a la plus petite croissance. La fertilisation est donc en corrélation avec la hauteur des plants ce qui est conforme aux résultats de Assigbé, Akakpo et al. (2005) qui ont rappelé que les engrais chimiques améliorent la croissance du riz.

Le traitement avec Azolla a donné la meilleure croissance donc A. pinnata permet une bonne croissance du riz. Celle-ci est possible en raison de la mise à disposition de façon permanente de l'Azote assimilable à la plante de riz par A. pinnata comme l'a montré les travaux de Maria Andrea et al. (2007). En effet grâce aux hétérocystes contenus dans les cellules de A. pinnata qui sont des sites fixatrices de l'azote 
atmosphérique, (Dupy et al., 1987), la plante de A. pinnata fixe et accumule de l'azote qu'elle transforme en ammoniaque. Cette forme d'azote assimilable permet la croissance des plants de riz. Comme en Asie et au Madagascar, A. pinnata est une plante capable d'augmenter la croissance du riz au Bénin. Cette constatation est conforme aux études de Becking (1979), qui préconise l'utilisation de A pinnata dans les zones tropicales pour limiter la pollution agraire causée par la culture du riz traité avec des engrais chimiques.

Enfin ces études montrent qu'une bonne croissance du riz est une des conditions sine qua non pour un très bon rendement du riz.

Effet de substitution des engrais chimiques par A. pinnata sur les rendements du riz

L'analyse statistique de ces résultats montre qu'il existe une différence hautement significative $(\mathrm{P}<0,01)$ entre les traitements à différentes doses d'engrais et le traitement sous fertilisation de $A$. pinnata en ce qui concerne les rendements de même, qu'ils ont une différence significative avec le témoin. Cela signifie que la fertilisation minérale augmente les rendements. Ces résultats sont en accords avec les travaux de Malavolta et Formasieni (1983), Barbosa (1987, 1991), qui affirment l'augmentation de la biomasse et de la masse du paddy avec l'utilisation d'engrais. Fageria et Baligar (2001) ont montré que la fertilisation augmente la production après un essai sur plusieurs variétés. Il en est de même pour les travaux de Mamadou Dio-Liande (1981) selon lequel les engrais chimiques doublent le rendement de riz dans la zone de plateau de Côte d'ivoire. Brohl et al. (1997), ont aussi montré que l'apport d'azote sous forme d'engrais augmente le rendement en paille et la masse de matière sèche. Ils corroborent ceux de Stone et al. (1979) qui ont obtenu une augmentation de la productivité par l'application de $60 \mathrm{~kg}$ d'azote par hectare. Mais ils diffèrent fondamentalement des résultats de Crusciol (1982) qui a trouvé que le taux des nutriments n'a pas affecté le développement des plants. Cette contradiction s'explique par la richesse des sols des sites de leur expérimentation. Le traitement 4 à $100 \mathrm{~kg}$ de NPK et $50 \mathrm{~kg}$ d'urée par hectare est le traitement optimal au lieu du traitement 3 à $150 \mathrm{~kg}$ de NPK et $75 \mathrm{~kg}$ d'urée par hectare ce qui est contraire aux travaux de Assigbé (2002) pour qui le traitement qui donne le meilleur rendement est le traitement $3(150 \mathrm{~kg}$ de NPK et $75 \mathrm{~kg}$ d'urée par hectare). Comme pour la croissance, on pourrait expliquer cette différence par les conditions hydriques un peu difficiles au cours de l'expérimentation. Notre étude montre également que, sous la fertilisation organique de A. pinnata: le rendement de grain, celui de paille et de la masse de matière sèche augmentent. Ceci est, en adéquation avec Dièye (2002) qui a fait une étude comparative entre les fertilisations de $A$. pinnata, de bouse de vache et des engrais chimiques et qui conclue que, $A$. pinnata appliqué à $30 \mathrm{~kg}$ par hectare en azote, permet des niveaux de rendement supérieurs comparés aux traitements en fertilisation chimiques. Ces résultats sont aussi conformes à ceux de Lumpkin-Plucknet (1982), qui en concluent que : A. pinnata est un engrais vert permettant l'augmentation sensible du rendement du riz. Cette augmentation de rendement a lieu à cause de la mise à disposition de façon permanente de l'Azote assimilable à la plante de riz comme l'a montré les travaux de Maria Andrea et al. (2007). Cette Azote assimilable permet la croissance et augmente le rendement comme le montre l'analyse des rendements à la récolte. Selon Razaf et al. (1988), les caractéristiques microscopiques de la symbiose de $A$. pinnata résident dans la présence de nombreux sites de fixation et de transformation de l'azote atmosphérique en de 
l'azote assimilable de façon permanente. La riziculture sans engrais chimiques ou la réduction sensible de l'utilisation des engrais chimiques est donc possible au Bénin. Ces résultats sont conformes à ceux de Diara 2000 qui ont montré que l'utilisation de $A$. pinnata augmente le rendement en grain au Sénégal. Ces travaux sont également en conformité avec ceux de Rakotonasolo (1988) qui affirme que A. pinnata améliore de façon significative le rendement du riz à Madagascar ce que confirme aussi Reynaud (1984), qui affirme, que dans la zone tropicale sèche l'utilisation de $A$. pinnata en riziculture est une prospective agronomique importante.

\section{Conclusion}

L'intensification de la riziculture au Bénin s'accompagne d'une forte utilisation d'intrants chimiques agricoles à prix très élevés. De plus, ces intrants tels que utilisés actuellement, polluent la nappe phréatique et peuvent générer des problèmes de santé.

A. pinnata utilisée en riziculture, permet l'accélération de la croissance, la foliation, la mise en place des thalles et des panicules puis l'augmentation du rendement du riz. Il peut donc se substituer valablement aux engrais chimiques qui sont très chers, non accessibles aux paysans pauvres.

En substituant la fertilisation minérale et chimique du sol avec la fertilisation organique par A. pinnata en riziculture, on obtient une bonne croissance et un rendement meilleur que ceux des diverses doses d'engrais chimiques minéraux. Son utilisation est donc conseillée aux riziculteurs pour augmenter le rendement de la culture du riz et diminuer le coût de production. A. pinnata constitue donc, une solution à la pollution chimique liée à l'utilisation des intrants du riz au Bénin et une perspective agronomique en zone tropicale sèche (Reynaud, 1984). Il serait cependant judicieux malgré la performance avérée des résultats obtenus, de poursuivre ces travaux en étudiant, l'effet de A. pinnata sur les variétés de riz cultivés au Bénin, le comportement des adventices du riz face à cette variété de Azolla, le degré de réduction de la pollution de A. pinnata en riziculture, l'effet de $A$. pinnata sur la toxicité ferreuse des bas-fonds pour la riziculture au Bénin.

\section{REFERENCES}

Adegbola PY, Singbo G. 2005. Impact de l'importation du riz sur la compétitivité et la rentabilité de la production nationale au Bénin. Programme PAPA de l'IN RAB.

Assigbe. 2003. Maîtrise de l'eau pour la culture du riz: adaptabilité écologique des variétés vulgarisées au Bénin, 4p.

Assigbe P, Akakpo C, Adjé TI, Hononta E, Adjo L. 2005. Mieux Produire le Riz Pluvial et de Bas- Fond. INRAB.

Brohl AR, Karaman RM, Aktas A, Savasli E, 1997. Effect of nitrogen and phosphorus fertilisation on the yield and nutrient status of rice crop grown on artificial siltation soil from the kelkit river. $T r . J$. Agriculture Forestry, 22: 585-592.

CIRAD-GRET. 2002. Mémento de l'Agronome. Cirad, Gret. : Montpellier ; France, en ligne, $\mathrm{N}^{\circ}$ ISBN : 2-86844-1297 (Gret) - 2-87614-522-7 (Cirad).

Crusciol CAC. 1998. Effeitos de laminas de àgua e da adubaçào mineral em dois cultivares de arroz de sequeiro sob irrigaçào por aspersào. Botucatu/ UNESPA/FSA, 129p.

Diara HF. 2000. L'utilisation d'Azolla comme engrais vert dans la riziculture ouest africaine ADRAO - station régionale de saint-louis, Sénégal, $10 \mathrm{p}$.

Doberman A, Fairhurst T. 2000. Nutriments Disorders and Nutriments Management. International Plant Nutrition Institute; $191 p$.

DPP/MAEP. 2004. Annuaire Statistique. DPP/MAEP: Cotonou, Bénin. 
Dupuy J, Rabeharisola, Fetlarison. 1987. Mesure de la fixation biologique d'azote par Azolla sp. - Résultat des expériences préliminaires. Recherche pour le Développement. Série Sciences Biologiques $\mathrm{n}^{\circ}$ 4: $239-251$.

Fageria NK, Baligar VC. 2001. Lowland Rice Response to Nitrogen Fertilisation (vol. 32). Taylor \& Francis publishrs; 14051429.

FAO. 1997. Elaboration d'un plan national de relance de la filière riz. Rapport définitif. Vol. 1 et 2, FAO Projet TCP/BEN/5613 (A), Cotonou, 1997.

FAO. 2001. Annuaires Statistiques: FAO.

FAO. 2004. Année internationale du riz2004, Vingt troisième conférence régionale pour l'Afrique, Johannesburg (Afrique du Sud), 1- 5 mars 2004.

FAO. 2004. Production Year Book (vol.58). FAO: Rome.

Gaur JP, Noraho N. 1995. Role of certain environmental factors one cadmium uptake and toxicity in spirodela polyrhiza and Azolla pinnata. Biomedical and Environmental Science, 8: 202-210.

Irebrul G. 2004. Rizicultures Asiatiques, enjeu écologique et économique à l'aube du $\mathrm{XX}^{\text {ème }}$ siècle, $15 \mathrm{p}$.

Lacharme M. 2001. Le plant du riz .Données morphologiques et cycle de la plante "Fascicule2", Mémento technique de riziculture.

Lumpkin TA, Plucknett DL. 1982. Azolla as green manure use and management in top production. Westview prerr/Boulder, Colorado 230, pill.map.ref

Maclean JL, Dave DC, Hardy B, Hettel GP. 2002. Rice Almanac. Los Banos, International Rice Research Institute: Philippines; West Africa Rice Developpement Association Bouaké: Cote d'Ivoire; International Center for Tropical Agriculture: Cali, Colombia;
Food and Agriculture Organisation: Rome, Italy; 253p.

Malavota E, FornastériFiho D. 1983. Nutriçào mineral da cultura do arroz. In Cultura do Arroz de Sequeiro Fatores Afetando a Produtidade, Erreira ME, Yamada T, Malavolta E (eds). Instituto da Potassa \& Fosfata: Piracicaba; 95-143.

Mamadou Dio Liandé. 1981. Effets Direct et Combiné des Engrais et de Meloidogyne Incognita sur le Riz Pluvial. Laboratoire d'Hématologie, ORSTOM.

Maria Andrea K, Paul LG, Vlek. 2007. Azolla, une technique d'amélioration de l'efficacité d'utilisation de l'azote. Agriculture \& développement rural, Zentrum fur Entwicklungsforschung (Centre d'études pour le développement) Bonn, Allemagne.

Noraho , Gaurs. 1995. Adsorption and uptake of cadmium by Azolla pinnata: kinetics of inhibition by pressed Biomedical Environmental and Science, 8: 149 -1557.

Patrick WMH, Mahapatra IO. 1968. Transformation and availability to rice of nitrogen and phosphorus in water logged sorls. Adv. Agram., 29: 323 - 359.

Pande HK, Tran VD, That TT. 1997. Systèmes améliorés de riziculture pluviale. FAO, Rome, 11p.

Raemaeker. 2001. Agriculture en Afrique tropical ED CIP. Bibliographie Royale Albert I, Bruxelles.

Rakotonasolo F, Schramm. 1988. Contribution à l'étude de l'utilisation de Azolla comme engrais vert en riziculture malgache. Recherche pour le Développement. Série Sciences Biologiques $\mathrm{n}^{\circ}$ 7: 10.

Razaf, Mahenina, Schramm M. 1988. Contribution à l'étude de certaines caractéristiques microscopiques de la symbiose de Azolla pinnata.

Reynaud PA. 1984. Ecophysiologie des cyanobactéries fixatrices d'azote libre on 
en symbiose (Azolla) dans la zone tropicale sèche; prospective agronomiques. Thèse d'Etat Université d'Aix Marseille II, Faculté des sciences de LUMINY.

Reynaud PA, Franche C. 1986. Azolla Pinnata var africana. In De la Biologie Moléculaire aux Applications Agronomiques. ORSTOM : Sénégal ; 1 15.p

Sadiki M, Hilali A. 1992. Recent developpement in biological Nitrogen Fixation Research in Africa. Fifth International Conference, Rabat, Morocco, 10p

Sawadogo A et S. Kiemde, 2002. Mise au point de methodes de lutte contre les nematodes parasites $\mathrm{du}$ riz,
INERA/Station de Farako Bobodioulasso. In Actes de la Seconde Revue Régionale de la Recherchre Rizicole, 9p.

Shiomi Kitoh. 1993. The growth and nitrogen fixing Azolla of in polluted Aquatic water. Botany, 46: 129-139.

Van Hove CHF, Diara, Godard P. 1983.. Azolla en Afrique de l'ouest /West Africa bill, Morovia. West Africa Rice Developpement Association, $53 \mathrm{p}$.

Verlinden E, Soulé BG. 2003. Etude de la filière riz au Bénin: Diagnostic. Plan d'action PADSE .pp102. SOFRECO.18

Wilson G, Al-Hamdani SH (1997). Effect of chromium and. Humic substances on selected Physiological responses of Azolla caroliniana. Amer. Fern. J., 87: 17-27. 